\title{
Author Index to Volume 28
}

\begin{tabular}{|c|c|}
\hline Albini $\mathrm{CH}, 209$ & Chantin C, 127 \\
\hline Alpan G, 437 & Chao CR, 83 \\
\hline Alter C, 54 & Chapman TE, 153 \\
\hline Aranda JV, 142 & Charpie P, 213 \\
\hline Arato A, 579 & Chow W, 549 \\
\hline Arbel E, 232 & Christakis DA, 101 \\
\hline Archer E, 235 & Christensen RD, 323 \\
\hline Arlazoroff A, 232 & Christie CM, 11 \\
\hline Armstead WM, 609 & Clark ES, 28 \\
\hline Arvilommi H, 582 & Clyman RI, 437 \\
\hline Asakura T, 54 & Coleman SD, 348 \\
\hline Aubourg P, 657 & Cooke A, 199 \\
\hline Azad MAK, 166 & Coopes BJ, 101 \\
\hline & Crawford JD, 213 \\
\hline Bagherzadeh A, 266 & Crigler $\mathrm{Jr}, \mathrm{JF}, 213$ \\
\hline Bairam A, 142 & Crissinger $\mathrm{KE}, 28$ \\
\hline Balan VK, 529 & Cromby $\mathrm{CH}, 38$ \\
\hline Baldwin HS, 425 & Crone RK, 101 \\
\hline Ballard PL, 190 & Cronin CMG, 111 \\
\hline Ball K, 469 & Cross AS, 667 \\
\hline Balsan MJ, 111 & Crowley Jr, WF, 213 \\
\hline Baron K, 603 & Cussen L, 361 \\
\hline $\begin{array}{l}\text { Bashan N, } 19 \\
\text { Bassett JM } 388\end{array}$ & Cvitanovic-Sojat L, 24 \\
\hline \multicolumn{2}{|l|}{$\begin{array}{l}\text { Bassett JM, } 388 \\
\text { Battaglia FC, } 652\end{array}$} \\
\hline Baum M, 663 & Dascalu V, 374 \\
\hline Beaufrere B, 147 & Daughaday WH, 394 \\
\hline Beauregard LJ, 514 & Davis DJ, 190 \\
\hline Beharry K, 142 & Davis GJ, 567 \\
\hline Beitins IZ, 514 & Deakers TW, 446, 451 \\
\hline Belik J, 603 & de Goeij JJM, 332 \\
\hline Berne RM, 348 & deLemos RA, 367 \\
\hline Bertini E, 247 & De Meirleir LJ, 247 \\
\hline Bhatia SJS, 383 & Dempsher DP, 394 \\
\hline Bieber LL, 158 & Deshmukh DR, 256 \\
\hline Bier DM, 394 & Deshmukh GD, 256 \\
\hline Bierman F, 247 & DeVivo DC, 247,536 \\
\hline Bihoreau MT, 646 & Dewit O, 502 \\
\hline Binding $A, 5$ & Dibba B, 502 \\
\hline Birch DG, 485 & Dijkstra M, 339 \\
\hline Birch EE, 485 & DiMauro S, 247, 536 \\
\hline Bissonnette JM, 83 & Dinger J, 599 \\
\hline Blanchard PW, 142 & Dionisi-Vici C, 247 \\
\hline Blizzard RM, 11, 213 & Doré M, 327 \\
\hline Bode $\mathrm{HH}, 514$ & Doubilet P, 383 \\
\hline Bodurtha JN, 1 & Douglas SD, 120 \\
\hline Boepple PA, 213 & Drachman DB, 542 \\
\hline Bonham JR, 38 & Dudman NPB, 496 \\
\hline \multicolumn{2}{|l|}{ Bonilla E, 536} \\
\hline \multicolumn{2}{|l|}{ Bory C, 127} \\
\hline Boulieu R, 127 & $\begin{array}{l}\text { Eaves LJ, l } \\
\text { Ejsenherg L } 4 \mathrm{H}\end{array}$ \\
\hline Bourgneres P-F, 657 & Eisenberg L, 411 \\
\hline Boyle DW, 94 & Eto Y, 473 \\
\hline \multicolumn{2}{|l|}{ Bracco CA, 567} \\
\hline \multicolumn{2}{|l|}{ Brown RH, 542} \\
\hline Buist NRM, 529 & Fabre M, 657 \\
\hline Busija DW, 609,613 & Fabrizi GM, 536 \\
\hline \multirow[t]{2}{*}{ Butte NF, 631} & Ferlic L, 631 \\
\hline & Ferrari MM, 652 \\
\hline & Ferrazzi E, 652 \\
\hline Cannata $J, 361$ & Ferré P, 646 \\
\hline $\begin{array}{l}\text { Cannata J, 361 } \\
\text { Capaldi R, } 549\end{array}$ & Fewell JE, 374 \\
\hline Capaldi R, 549 & Filyk SC, 374 \\
\hline Capaldi RA, 529 & Firsching K, 379 \\
\hline Carpenter KH, 38 & Fisher DA, 622 \\
\hline Carrero-Valenzuela RD, 529 & Fogo A, 270 \\
\hline Cartier N, 657 & Fouke JM, 367 \\
\hline Cavill IAJ, 199 & Fröhlich $\mathrm{O}, 587$ \\
\hline \multirow{2}{*}{\multicolumn{2}{|c|}{ Cha C-JM, 641}} \\
\hline & \\
\hline Chance B, 54 & Galgani JP, 394 \\
\hline
\end{tabular}

Albini $\mathrm{CH}, 209$

Aranda JV, 142

Arato A, 579

Arbel E, 232

Arlazoroff A, 232

Asakura T, 54

Aubourg P, 657

Bagherzadeh A, 266

Bairam A, 142

Balan VK, 529

Ballard PL, 190

Ball K, 469

Balsan $\mathrm{MJ}, 111$

Bashan N, 19

Bassett JM, 388

Battaglia FC, 652

Baum M, 663

Beauregard LJ, 514

Beharry K, 142

Bertini E 247

Bhatia SJS, 383

Bieber LL, 158

Bierman F, 247

Bihoreau MT, 646

Binding A, 5

Bissonnette JM, 83

Blanchard PW, 142

Bode $\mathrm{HH}, 514$

Bodurtha JN,

Boepple PA, 213

Bonilla E 536

Bory C, 127

Boulieu R, 127

Bourgneres P-F, 657

Boyle DW, 94

Brown RH, 542

Buist NRM, 529

Busija DW, 609, 613

Campbell DE, 120

Cannata J, 36

Capaldi RA 529

Carpenter KH, 38

Cartier N, 657

Chance B, 54

\author{
Chantin C, 127 \\ Chao CR, 83 \\ Chapman TE, 153 \\ Chow W, 549 \\ Christakis DA, 101 \\ Christie CM, 11 \\ Coleman SD, 348 \\ Cooke A, 199 \\ Cote A, 218 \\ Crawford JD, 213 \\ Cromby $\mathrm{CH}, 38$ \\ Crone RK, 10 \\ Cronin CMG, 111 \\ Crowley Jr, WF, 213 \\ Cussen L, 361 \\ Dascalu V, 374 \\ Daughaday WH, 394 \\ Deakers TW, 446, 451 \\ Goeij JJM, 332 \\ De Meirleir LJ 247 \\ Dempsher DP, 394 \\ DeVivo DC, 247, 536 \\ Dewit O, 502 \\ Dijkstra M, 339 \\ DiMauro S, 247, 536 \\ inger J, 599 \\ Doré M, 327 \\ Doubilet P, 383 \\ Dudman NPB, 496 \\ Eaves LJ, 1 \\ Eisenberg L, 411 \\ Eto $\mathrm{Y}, 473$ \\ Fabre M, 657 \\ Fabrizi GM, 536 \\ Ferlic L, 631 \\ Ferrazzi E, 652 \\ Ferré P, 646 \\ Firsching K, 379 \\ sher DA, 622 \\ Fogo A, 270 \\ Fröhlich O, 587 \\ Galgani JP, 394
}

Gallaher KJ, 455

Galler JR, 235

Garty B-Z, 120

Garza C, 63

Gasser T, 5

Gehrhardt B, 79

Gelardi NL, 641

George JW, 567

Gershan WM, 87

Gerstmann DR, 367

Gest AL, 223, 613

Ghishan FK, 591

Gibson KM, 518

Giesler M, 613

Girard J, 646

Gleason CA, 50

Glerum DM, 549

Gluckman PD, 469

Gmyrek D, 79, 599

Goldberg M, 232

Gonzales J, 507

Gonzales LK, 190

Granger DN, 28

Granger JP, 460

Grumbach MM, 562

Grummer MA, 186

Gunn RB, 587

Haddad GG, 218

Halliday D, 240

Hamaoka K, 429

Hansen TN, 75, 223, 613

Hanson C, 388

Harris MC, 120

Havinga R, 339

Headrick JP, 348

Heath-Monnig E, 394

Hendrickx AG, 567

Henry JF, 176

Hershenson MB, 101

Hershfield M, 127

Hewitt JK, 1

Hirst K, 94

Hoffman DR, 485

Hohimer AR, 83

Holland BM, 199

Holme E, 131

Howells DW, 116

Hoy TG, 199

Hudson IRB, 199

Hull D, 38

Hurko O, 542

Hurwitz RE, 261

Hutchens TW, 176

Hyland K, 116

Ibata Y, 429

Ichikawa I, 270

Ida $\mathrm{H}, 473$

Iden $\mathrm{P}, 518$

Ijlst L, 657

Ikoma M, 270

Isolauri $\mathrm{E}, 31,582$

Jacobi MS, 87

Jacobs MM, 190

Jalonen T, 582

Jame P, 646

James LS, 595

Jansson T, 203
Jarriel WS, 75

Jensen MC, 394

Jobe AH, 313

Johns DR, 542

Joiner $\mathrm{CH}, 587$

Jones $\mathrm{Jr}, \mathrm{MD}, 50$

Kalamaras JA, 469

Katz S, 1

Kaufmann B, 667

Kawai H, 316, 572

Kennaway NG, 529

Kerner J, 158

Kerrigan JR, 11

Kerzner B, 227

Khoury J, 493

Kikuchi N, 401, 405

Kim KS, 667

Kim SU, 473

Kiu GT, 595

Klein PD, 631

Knopp SJ, 83

Kodama M, 42

Kohelet D, 232

Komeda T, 429

Komiyama A, 316

Kondo CS, 374

Koster JF, 344

Kretchmer N, 261

Kristiansson B, 131

Kroos MA, 344

Ktorza A, 646

Kuipers F, 339

Kuribayashi T, 429

Kursar JD, 379

Lafeber HN, 153

Lambert R, 336

Landy $\mathrm{H}, 213$

Largo RH, 5

Larsson N-G, 131

Laudignon $\mathrm{N}, 142$

Lebenthal E, 158, 166

Lee CP, 542

Leffler CW, 609, 618

Levinsky RJ, 116

Lezza AMS, 525

Liechty KW, 323

Lifshitz F, 31

Lightowlers R, 529, 549

Light RB, 603

Link K, 213

Lloyd TR, 425

Lombes A, 536

Loonen MCB, 344

Lott MT, 525

Loughead JL, 493

Ludomirsky A, 120

Lueder FL, 464

MacGillivray MH, 209

MacGregor DN, 63

Mädler H-J, 79

Maesaka H, 401, 405

Magnuson JS, 176

Mannelli SC, 469

Mansfield MJ, 213

Marconi AM, 652

Martens ME, 542

Martha Jr, PM, 11 
Martin CG, 223

Matherne GP, 348

Mathieu M, 127

Matsuda I, 42

Matsuoka T, 24

Mauras N, 626

Mauray F, 437

Mayevsky A, 54

Mayock DE, 196

McClung HJ, 227

McNerney ME, 106

Meert K, 256

Merola AJ, 227

Meschia G, 94

Middleton B, 518

Miller NR, 542

Milley JR, 111

Mills BJ, 209

Mimouni F, 493

Miranda AF, 536

Mirro R, 609, 613

Mita S, 536

Mitchell HW, 69

Mizuta T, 429

Moessinger AC, 595

Moïse AA, 223

Moran A, 19

Morley DS, 235

Moscicki A-B, 507

Moses SW, 19

Moskowitz WB, 1

Mosteller M, 1

Mullahoo K, 142

Murphy AM, 46

Murray R, 227

Musjce DJ, 354

Nagaoka A, 429

Nakagawa M, 429

Nakamura R, 42

Nakamura T, 429

Nakase H, 536

Nance WE, 1

Neilsen NR, 327

Newth CJL, 446, 45I

Nioka $S, 54$

Nishiyama $S, 42$

Noguchi A, 379

North D, 622

Notoroberto NF, 83

Nurjhan N, 646

Ogata ES, 464

Ohta T, 42

Oh W, 641

Oldfors A, 131

Olson AL, 183

O'Rourke PP, 101

Øyasæster S, 477, 482

Pace WR, 75

Pachiaudi C, 147
Pacy PJ, 240

Page LA, 514

Pagon RA, 247

Palefsky J, 507

Pardi G, 652

Peleg N, 19

Penn D, 158

Perlman S, 183

Persson E, 203

Peterson PL, 542

Petrova-Benedict R, 549

Phibbs RH, 567

Picon L, 646

Plappert T, 383

Polin RA, 120

Pollack JD, 227

Pollitt RJ, 38

Potashnik R, 19

Poulsen JP, 477, 482

Pourcyrous M, 618

Powell BR, 529

Powers P, 227

Prader A, 5

Prentice A, 502

Pulver P, 247

Putet G, 147

Quattrin T, 209

Quigley R, 663

Ramsey FC, 235

Rannels DE, 455

Rattenbury JM, 38

Reddy R, 379

Reilly MP, 54

Reuser AJJ, 344

Rhead WJ, 524

Riby JE, 261

Richards GE, 469

Richardson P, 75

Richmond JB, 415

Roberts B, 31

Roberts JM, 190

Robillard JE, 183

Robinson BH, 518, 549

Rocchiccioli F, 657

Rognum TO, 477, 482

Rogol AD, 11, 626

Rolland MO, 127

Romero G, 213

Rossi TM, 158

Rotwein PS, 394

Royce PM, 137

Rutledge SL, 542

Ryan JP, 336

Sadoff J, 667

Said HM, 266

Salle B, 147

Saltzman DH, 383

Sanderud J, 477

Sauer PJJ, 153
Saugstad OD, 477, 482

Savilahti E, 579

Schaefer F, 5

Schaller P, 79, 599

Schärer K, 5

Schieken RM, 1

Schmidt-Sommerfeld E, 158

Schoenfeld DI, 213

Schon EA, 536

Schoolnik GK, 507

Schulze A, 79, 599

Seibel P, 525

Seidel C, 5

Servidei S, 247

Shannon KM, 567

Sharifian A, 266

Shaw MD, 11

Shen CT, 595

Sherwood WG, 518

Shibata H, 591

Shimoo K, 429

Shoffner JM, 525

Short BL, 50

Shulman RJ, 171

Singh KR, 256

Sivan Y, 446, 451

Slauson DO, 327

Sloan HR, 227

Smit EP, 339

Smith CV, 613

Smith DS, 54

Smith EO, 631

Smith HW, 613

Smith I, 116

Smith IL, 567

Smith JB, 120

Solaro RJ, 46

Solhaug MJ, 460

Souillet G, 127

Søvik O, 518

Sparrow MP, 69

Standaert TA, 196

Statement JPE, 561

Steinmann B, 137

Stephenson TJ, 38

Stewart ML, 361

Stine OC, 542

St. John Sutton M, 383

Strobel S, 116

Styne DM, 15

Sugama S, 473

Sulkers EJ, 153

Suwa S, 401, 405

Sweetman L, 518

Szeto $\mathrm{HH}, 106$

Szymonowicz W, 361

Tachibana K, 401, 405

Tagliaferri RP, 69

Taylor AF, 367

Taylor BJ, 374

Teichberg S, 31

Tein I, 247
Thach BT, 87

Theard MA, 383

Thompson GN, 240

Tollefsen SE, 394

Towfighi J, 354

Traystman RJ, 50

Trevett D, 199

Tritschler H-J, 536

Trivedi B, 394

Tsang RC, 493

Tulinius M, 131

Turner TL, 199

Tyson JE, 485

Uauy RD, 485

Usher M, 232

Valdes E, 75

Van Den Hamer CJA, 332

van der Kraaij AMM, 344

van der Ploeg AT, 344

Vannucci RC, 354

Van Wouwe JP, 332

Veldhuis JD, 626

Veldhuizen M, 332

Verkasalo M, 579

Vianey-Liaud C, 657

Virtanen E, 582

Voljavec AS, 525

Vonk RJ, 339

Walker AM, 361

Walker LK, 50

Wallace DC, 525

Wallace MR, 460

Wanders RJA, 657

Wang XL, 496

Wapnir RA, 31

Wardrop CAJ, 199

Wassner S, 455

Watchko JF, 196

Watts RWE, 240

Weeding CM, 388

Wheeler MD, 15

Wilcken DEL, 496

Wilkening RB, 94

Willemsen R, 344

Winkler U, 599

Winter DC, 367

Wolpert E, 455

Wong WW, 631

Woodrum DE, 196

Yabuhara A, 316, 572

Yip T-T, 176

Yoshioka T, 270

Yu VYH, 361

Zachman RD, 186

Zerbe GO, 94

Zhang Y-Z, 529

Ziff EB, 63

Zollinger W, 667 